\title{
The Influence of Air Flow Velocity and Particle Size on the Collection Efficiency of Passive Electrostatic Aerosol Samplers
}

\author{
Ramin Jajarmi Imani ${ }^{1}$, Laila Ladhani ${ }^{2}$, Gaspar Pardon ${ }^{2}$, Wouter van der Wijngaart ${ }^{2}$, \\ Etienne Robert ${ }^{3 *}$ \\ ${ }^{1}$ Department of Mechanics, KTH Royal Institute of Technology, Stockholm, Sweden \\ ${ }^{2}$ Department of Micro and Nanosystems, KTH Royal Institute of Technology, Stockholm, Sweden \\ ${ }^{3}$ Department of Mechanical Engineering, Polytechnique Montréal, Montréal, Québec, Canada
}

\begin{abstract}
Electrostatic sampling is a promising method for the collection of bioaerosol particles. Although the underlying physics responsible for particle collection are well understood, the collection efficiency of simple passive electrostatic samplers is difficult to predict. Under these conditions, the collection efficiency becomes very sensitive to ambient air current and particle size, especially for submicron particles relevant for airborne virus transmission. In this paper, we compare two electrostatic aerosol sampler designs, a commercial product consisting of a flat collector plate located in the same plane as the charging needles and an axisymmetric design sampling directly to a liquid droplet. The aerosol particle collection efficiency of the samplers is investigated for particle size ranging from 0.25 to $2 \mu \mathrm{m}$ while the air flow velocity surrounding the samplers is varied from 0.3 to $1 \mathrm{~m} \mathrm{~s}^{-1}$. For the planar design, at all ambient flow velocities, the submicron fraction of the particles captured originates in streamlines up to a maximum of $75 \mathrm{~mm}$ above the surface of the device collector, which greatly limits the volume of air being effectively sampled. The axisymmetric design features a nonmonotonic capture efficiency as a function of particle size, with a minimum between 0.4 and $0.8 \mu \mathrm{m}$. The flow field in the inter-electrode region, captured using particle image velocimetry (PIV) reveals the presence of strong recirculation zones that can be responsible for the increased collection efficiency for very small particles.
\end{abstract}

Keywords: Electrostatic precipitation; Submicron particles; Quantitative; Sampling; PIV.

\section{INTRODUCTION}

Bioaerosols are found nearly everywhere in both indoor and outdoor environments and they can affect the health of humans (Walser et al., 2015), animals (Van Leuken et al., 2016) and plants (Brown and Hovmøller, 2002). Means for measuring the concentration and composition of bioaerosol particles are crucial for understanding aerosol evolution, identifying its source, assessing exposure levels, and monitoring control measures (Kulkarni et al., 2011). Recent threats of bioterrorism and recurring infectious disease epidemics have renewed interest in environmental bioaerosol sampling, specifically with the objective of airborne pathogen detection. Although there is no lack of appropriate analysis techniques, challenges remain for the reliable collection of relevant bioaerosols particles, especially those involved in virus transmission, due to their typically extremely low concentration, small size and fragility. For instance,

\footnotetext{
* Corresponding author.

E-mail address: etienne.robert@polymtl.ca
}

measurement of airborne norovirus in clinical settings by Bonifait et al. (2015) have revealed a concentration of between and genomes per $\mathrm{m}$. Under those conditions, the mass of interest for airborne virus detection is negligible relative to the rest of the particles in typical indoor and outdoor aerosols. This can be challenging for the development of novel real-time or near real-time detection tools based on microfluidic approaches sensitive to sensor fouling. Knowing the size-dependence of the sampler collection efficiency therefore becomes a necessity to be able to design them for specific size ranges and thus avoid diluting the target with the rest of the suspension (Alonso et al., 2015).

The most commonly used methods for bioaerosol sampling are either based on filtration or inertial impaction on surfaces (Verreault et al., 2008). Electrostatic precipitation (ESP), a technique already well-known for cleaning industrial gas streams, has also been investigated for sampling airborne pathogens (Gerone et al., 1966; Kulkarni et al., 2011). This method brings interesting features, such as low mechanical stress on the particles collected, i.e. the deposition velocity is much lower compared to approaches based on inertial impaction (Willeke et al., 1995), meaning that mechanical stress is less likely to affect the infectivity of the pathogens 
collected. However, when assessing the effect of ESP sampling on the infectivity of the pathogen collected, the effect of ozone production needs to be considered (Ning et al., 2008). Another sought feature for bioaerosol sampling that can be provided by ESP is the ability to send particles directly to liquid microfluidic systems, allowing for fielddeployable real-time or near-real-time biological detection assays (Pardon et al., 2015). ESP samplers are also capable of treating large volumes of air with low power consumption, low pressure drop and no risk of clogging (Kettleson et al., 2013). Besides these potential benefits, ESP samplers have limitations that need to be characterized and addressed to yield useful results.

Sampler designs that rely on a mechanical pumping to circulate the aerosol in the collector region have received more attention relative to passive systems (Han et al., 2015; Park et al., 2015) and information on the size-dependence can generally be calculated from the known flow field. Although the pumping mechanism allows the precise determination of the sampling location and volumetric flow rate, the overall complexity of such samplers reduces their usefulness in some applications. In clinical settings for instance, silent and maintenance-free operation are key advantages.

In this paper, we investigate passive ESP samplers that do not rely on external pumping mechanisms to bring an aerosol stream between the electrodes. The motion of the fluid is rather caused by the electrical field itself, resulting in a design attractive for its simplicity and robustness. However, with this approach the collection efficiency becomes very sensitive to the particle size and the velocity of ambient air currents surrounding the sampler. Passive ESP samplers are already being used in medical studies to capture indoor aerosols, unfortunately often without sufficient consideration for these parameters (Parvaneh et al., 2000; Hagbom et al., 2015). For experimental results obtained with these samplers to be interpreted correctly, the effect of both parameters on the collection efficiency needs to be carefully assessed, keeping in mind that it will depend strongly on the geometry of the ESP electrode configuration. The objective of the present study is therefore to quantify the collection efficiency of different passive ESP sampler designs in variable air currents, allowing users to estimate the size selectivity of their device and the effect of ambient air currents. Although a broad range of particle sizes are relevant to bioaerosol sampling problems (Lighthart and Shaffer, 1997), we focus our investigation on the sub-micron range, relevant for instance in airborne virus transmission. We expect the effect of the two critical parameters mentioned above to be most significant in this range, for which the collection efficiency of passive ESP samplers has moreover rarely been characterized quantitatively.

In ESP samplers, particles only acquire a charge, and therefore a force to migrate towards the collection electrode, if they remain for a sufficiently long time in a sufficiently strong electrical field. Because the fluid motion induced by passive ESP samplers is typically on the same order of magnitude as indoor air currents, the residence time of particles in the field can vary greatly in practical applications.
Such bulk motion of the air can for instance be caused by ventilation systems, thermal convection or personnel movements and makes it much harder to capture small aerosol particles on surfaces as they have a natural tendency to follow streamlines. The effective volumetric aerosol flow rate effectively affected by a given electrostatic field geometry and strength is therefore dependent on the average fluid velocity surrounding the sampler. More importantly, this velocity also affects the distance that particles can travel to reach the collection electrode, defining the location sampled in a flow field. This information is of critical importance for instance when selecting where to position a passive ESP sampler to characterize the aerosol present in a room.

Several electrostatic sampler designs have been developed in recent years specifically to capture particles in bioaerosols. Comparison with more established sampling tools is now available (Dybwad et al., 2014) for a few designs, but quantitative size-dependent capture efficiency is scarce (Han and Mainelis, 2008; Sillanpää et al., 2008). Understanding the effect of particle size and surrounding air currents on the performance of the passive ESP samplers necessitates quantitative information on the collection efficiency obtained under controlled conditions where the flow field is known precisely, along with the size and number of particles removed from the flow. Here we provide this information for two passive ESP sampler geometries, one planar and one axisymmetric. In the former the charging and collection electrodes are in the same plane and the sampled particles are deposited on a solid surface. It is a commercial product, Ion-Flow Ionizing Technology from LightAir AB (Airpoint), used previously by Hagbom et al. (2015) to perform an airborne virus infectivity study. The latter is a recent development by Pardon et al. (2015) intended for point-of-care (PoC) near-real-time detection systems, sampling particles directly to a microfluidic interface. It features a cylindrical design and is referred to as the ElectroStatic Sampler for Aerosol (ESSA). The results also include a detailed characterization of the flow field around the samplers gathered using Particle Image Velocimetry (PIV) and Laser Doppler Anemometry (LDA).

\section{EXPERIMENTAL SETUP}

The investigation is carried out by fixing the ESP samplers in a low speed wind tunnel and seeding the flow around it with a polydispersed submicron aerosol. The collection efficiency is estimated as a function of particle size and ambient air velocity by measuring the concentration and size distribution of the aerosol particles downstream of the collector. The experimental equipment and methods are further described in the following sections.

\section{Electrostatic Samplers}

The first ESP sampler investigated collects particles on a flat surface, with charging electrodes located in the same plane. It is a commercially available instrument (Airpoint Sverige $\mathrm{AB}$ ) that operates at $-17 \mathrm{kV}$, generating a negative corona using 3 needles as discharge electrodes, labeled as 
a)

Wind tunnel
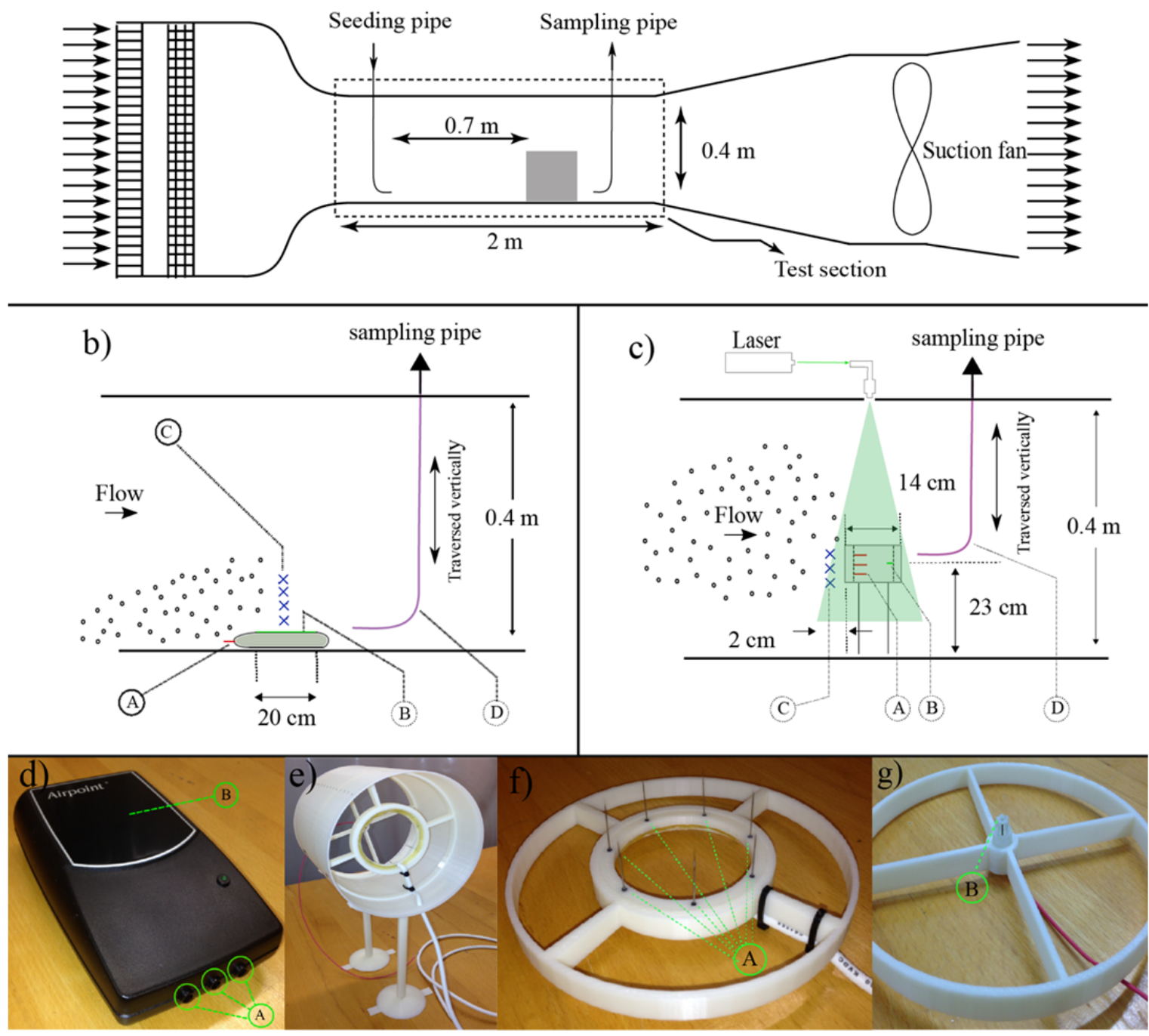

Fig. 1. (a) Schematic representation of the open-circuit wind tunnel, the shaded gray box representing the location where velocity measurements are conducted around the samplers. (b) and (c) magnified view of the Airpoint and ESSA samplers in the test section. Label: (A) negative electrode, (B) positive electrodes, (C) LDA velocity measurement points. (d) Airpoint ESP sampler. (e)-(g) ESSA Sampler, negative electrodes and positive electrode, respectively.

A in Fig. 1(b)). A stainless-steel plate $88 \mathrm{~mm} \times 100 \mathrm{~mm}$ $(\mathrm{W} \times \mathrm{L})$ is used as the collection electrode, and is set to the ground potential. The electrodes are housed in a plastic case. For safety reasons, the current at the collector is kept very low, below $80 \mathrm{~mA}$.

The other passive ESP sampler is referred to as the ESSA (ElectroStatic Sampler for Air), shown in Fig. 1(e)), a recent development featuring a axisymmetric cylindrical design to increase the collection efficiency of fine bioaerosol particles. It consists of a cylindrical shell (open at both ends), $140 \mathrm{~mm}$ in diameter and $140 \mathrm{~mm}$ in length. Two 3D-printed skeleton support structures sit tightly inside the shell and house the discharge and collection electrodes. The discharge electrode consists of seven tungsten needles, with a tip radius $<10 \mu \mathrm{m}$, placed axisymmetrically at a radius of $32.5 \mathrm{~mm}$ from the shell axis. The collection electrode is an electrically-connected $200 \mu \mathrm{L}$ saline liquid droplet held in place by surface tension using a specially designed cage collector. This cage collector is intended to maximize the area of the air-to-liquid interface while minimizing air-tosolid interface, to capture airborne particles directly into liquid, allowing for convenient integration with $\mathrm{PoC}$ analysis systems and direct bio-analysis of airborne pathogens (Ladhani et al., 2015). In this configuration, the electric field is optimally converging towards the collector, enabling efficient and localized sampling. Unlike the Airpoint, the ESSA design allows independent control of the interelectrode distance and applied potential difference. For the results presented here, this potential difference was varied between $13 \mathrm{kV}$ and $17 \mathrm{kV}$.

\section{Wind Tunnel and Diagnostic Tools}

The experimental setup, shown schematically in Fig. 1, is composed of an open-circuit wind tunnel that provides a 
controlled air flow around the samplers, an aerosol generator, measurement instrumentation and the electrostatic samplers under investigation. The wind tunnel has a rectangular test section with dimensions of $0.40 \mathrm{~m} \times 0.50 \mathrm{~m}$ $\times 2 \mathrm{~m}(\mathrm{~W} \times \mathrm{H} \times \mathrm{L})$. The air velocity range was chosen to cover the conditions encountered in typical indoor settings: 0.3-1 $\mathrm{m} \mathrm{s}^{-1}$ (Baldwin and Maynard, 1998). To reveal the amount and sizes of the particles captured, a small proportion of the flow is removed downstream of the samplers and analyzed using a particle spectrometer (MiniLAS 11-R, GRIMM). An atomizer (ATM221, TOPAS) is used to generate polydispersed aerosols from Di-EthylHexyl-Sebacat (DEHS) oil, or $\mathrm{KCl}$ in aqueous solution, first atomized then dried in a desiccation column. Both aerosols were measured to have a stable particle number density and size distribution within $\pm 10 \%$ over 30 minutes of operation. For practical reasons, the entire volume of air flowing through the wind tunnel could not be seeded. Aerosols are inserted in the mid-plane of the test section through a L-shaped stainless steel pipe, with $5 \mathrm{~mm}$ inside diameter, $70 \mathrm{~cm}$ upstream of the location of the samplers. The sampling pipe is also positioned at the mid plane and can be traversed vertically using a micrometer translation stage, as shown in Fig. 1.

Knowledge of the flow structures present around the samplers is paramount to the interpretation of the collection efficiency data. For this reason, two optical flow measurement techniques were implemented: Particle Image Velocimetry (PIV) and Laser Doppler Anemometry (LDA). PIV allows for the visualization of large scale structures while LDA was used to gather precise velocity profiles around the collection electrodes of the samplers. The PIV system allowed the measurement of the flow with a field of view $40 \times 55 \mathrm{~mm}$ using a high-speed camera (LaVision FlowMaster). The LDA system (Dantec Dynamic FlowLite) provides velocity data with high temporal resolution from a measurement volume approximately 0.75 . Both techniques rely in the presence of tracer particles in the flow. As these particles are Lagrangian tracers, they will follow the streamlines of the flow in the absence of external forces. Consequently, the velocity results gathered when the electric field is present should carefully be interpreted as the particle velocity vector are not necessarily representative of flow streamlines.

\section{Data Acquisition}

The particle number density and size distribution are measured downstream of the samplers using the optical spectrometer every 6 seconds. Data is acquired by keeping the sampling tube at a fixed location and turning the ESP samplers on and off at time intervals of 2 minutes ( $50 \%$ duty cycle), for a total of 20 minutes. To assess the collection efficiency of the samplers for particles of different sizes, the distributions are compared through a size-dependent figure of merit $(\eta)$ using the particle number density measured for each size bins during periods where the electric field was on and off. This collection efficiency for different particle diameter size bins $\left(d_{p}\right)$ is defined as the difference between the averaged particle number density in two consecutive intervals (i) without and with electrical field, $C_{o f f, i}$ and $C_{o n, i}$, divided by $C_{o f f, i}$. It is then averaged over consecutive onand-off sequences over a 20-minute acquisition time, as shown in Eq. (1).

$$
\eta=\frac{\sum_{i}^{n} \frac{C_{d_{p i} i}^{o f f}-C_{d_{p} i}^{o n}}{C_{d_{p} i}^{o f f}}}{n}
$$

Here, $\mathrm{n}$ is the number of the on-and-off intervals during the 20-minute sampling time. Positive values of $(\eta<0)$ therefore indicate that when electrodes are electrically charged particles are either captured by the samplers or migrated away from the sampling pipe. In other words, $\eta$ represents the relative change in the particle number density for each size bin. This also implies that particle deposition on the non-collecting surfaces of the samplers is interpreted as capture of particles. Although this is a source of uncertainty in the measurements presented, we believe that the error caused by this effect is small as the area of such non-collecting surfaces is minimized and the material used is not electrically conductive.

\section{RESULTS AND DISCUSSION}

\section{Air Velocity Profile at the Samplers}

1D velocity profiles above the Airpoint with and without the electric field, obtained using the LDA system, are shown in Fig. 2 for a case where the wind tunnel provides an averaged bulk velocity around the sampler of $\simeq 0.39 \mathrm{~m} \mathrm{~s}^{-1}$. These results shown in Fig. 2(a)) are obtained for the streamlines immediately above the collector surface of Airpoint sampler, with the flow continuously seeded with DEHS oil particles with a mean diameter of $300 \mathrm{~nm}$. For this relatively low convection rate, only small changes in the axial velocity above the Airpoint are observed: the particles are decelerated in a layer of fluid with a thickness of approximately $2 \mathrm{~cm}$ and accelerated above that. These profiles reveal that submicron DEHS oil particles flowing more than $6 \mathrm{~cm}$ above the collection surface are largely unaffected by the presence of the electric field.

Similar velocity profile gathered around the ESSA reveal a much stronger influence of the electric field on the trajectories of the particles, as can be seen in Fig. 2(b)). The measurement is conducted across the diameter of the shell in its mid-plane and $2 \mathrm{~cm}$ upstream of its entrance, both in the presence and in the absence of electrical charging. These profiles show the occurrence of an unexpected and strong back-flow along the axis of the cylindrical shell when the electric field is present.

To shed light on this phenomenon, PIV was used to visualize flow structures in the inter-electrode region and close to the collecting electrode. The use of a transparent cylindrical shell allowed optical access to the inner volume of the ESSA and the flow was seeded with DEHS oil particles to maximize laser light reflections. Time-averaged velocity fields in the inter-electrode region for cases with and without electrical field are shown in Fig. 3. Fig. 3(a)) 

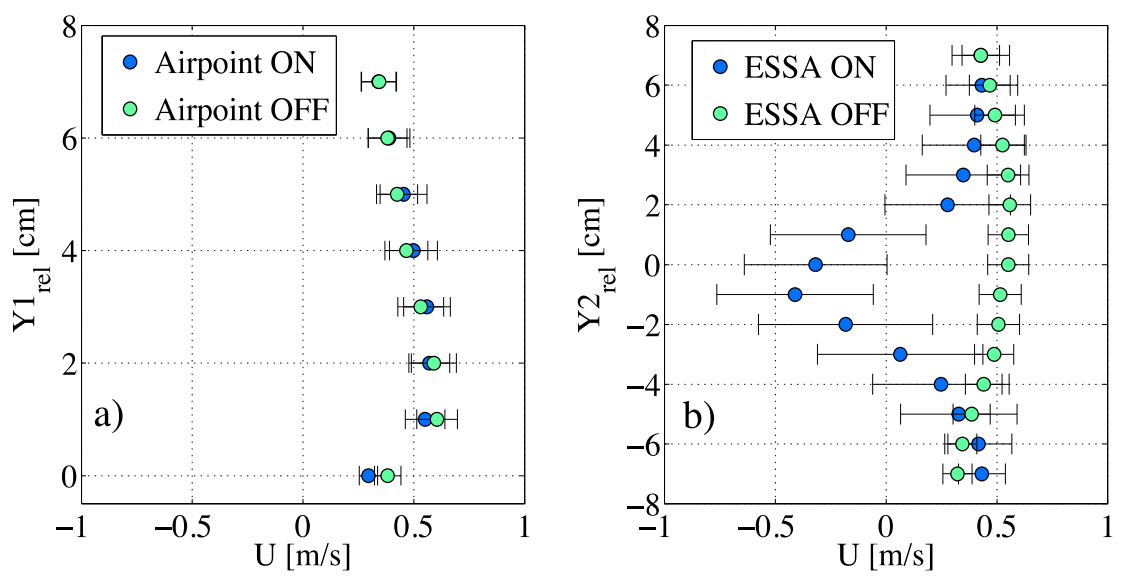

Fig. 2. (a) Velocity profile above the Airpoint collection surface with the relative distance from the collection surface. (b) Velocity profile $2 \mathrm{~cm}$ upstream of the ESSA with) the relative distance to the axis of the cylindrical shell. The wind tunnel center velocity is $\cong 0.39 \mathrm{~m} \mathrm{~s}^{-1}$.

a)

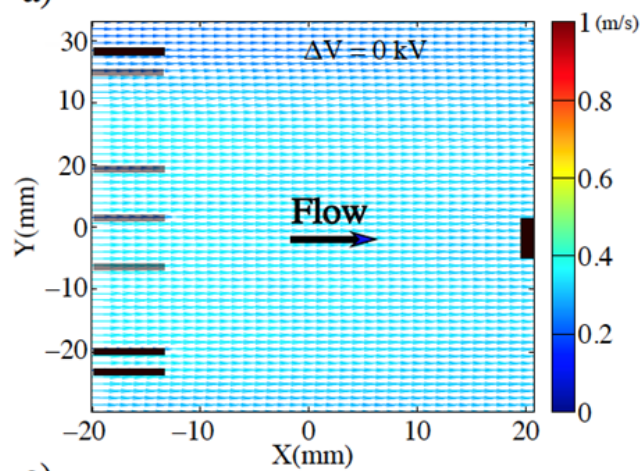

c)

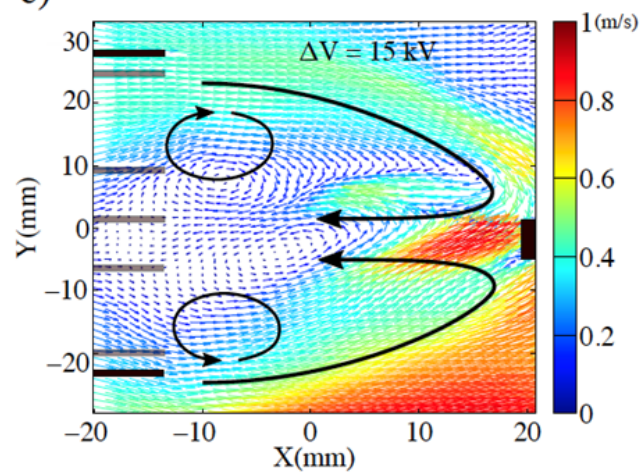

b)

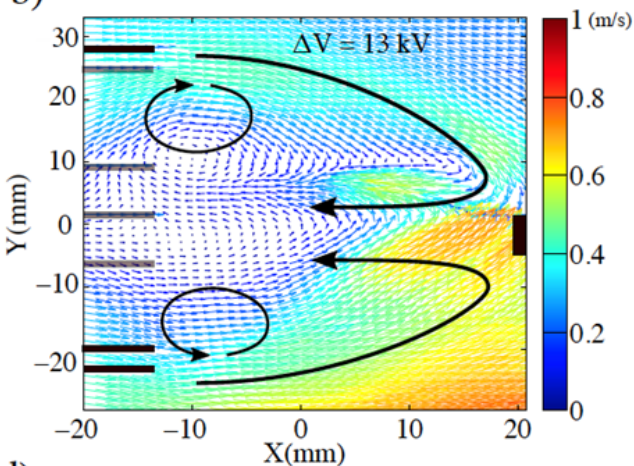

d)

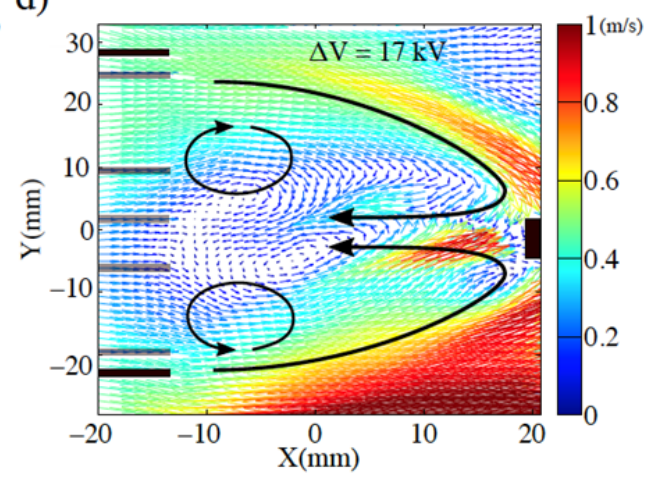

Fig. 3. Time-averaged velocity fields measured by PIV in the inter-electrode region at the mid-plane of the ESSA sampler. Color scale represents the magnitude of velocity in $\mathrm{m} \mathrm{s}^{-1}$. (a) Without the electrical field. (b)-(d) With electric fields having a $13 \mathrm{kV}, 15 \mathrm{kV}, 17 \mathrm{kV}$ potential difference, respectively. The needles on the left side of figures (b), (c), (d) are charged negatively with $8 \mathrm{kV}, 9 \mathrm{kV}, 10 \mathrm{kV}$ respectively, while the collector is charged positively with $5 \mathrm{kV}, 6 \mathrm{kV}, 7 \mathrm{kV}$, respectively. Not all needles are in the laser plane for PIV measurement.

shows a uniform velocity field across the ESSA in absence of the electrical field. Figs. 3(b)-3(d)) show the time-averaged velocity fields for cases with $13 \mathrm{kV}, 15 \mathrm{kV}, 17 \mathrm{kV}$ potential differences, respectively. In the presence of an electric field, convective cells are detected in the inter-electrode space. As the magnitude of the electric potential imposed between the electrodes is increased the migration speeds of the oil particles also increases, as seen in Figs. 3(b)-3(d)). In these cases, two convective rolls can be seen in the measurement plane, from the negative electrodes towards the positive collector. These rolls result from the convergence of the 7 streams emanating from the needles towards the collector, forming a cone-shaped structure. As these jets meet at a single point, part of the momentum is directed backward, forming the back-flow observed along the axis of the cylinder. With higher electrical potential difference 
the recirculating features becomes stronger in the interelectrode region due to a higher convection of mass towards the collector, as shown in Figs. 3(b)-3(d)).

The higher particle speeds observed at the bottom of the ESSA is likely due to a smaller azimuthal distance between the nearest needle and the laser sheet at this location compare to the configuration at top. As a result, the velocity field captured in the plane of the laser sheet reveals a higher magnitude around the convective stream emanating from this needle in the lower part of the image. The other needles also generate their own air streams, however they are not visualized here since they do not fall in the plane of the laser sheet.

\section{Collection Efficiency: Effect of Particle Size and Residence Time}

The collection efficiencies of the Airpoint and ESSA samplers as a function of particles diameter and air velocities are studied using the merit defined in Eq. (1). The particles used are dried droplets of KCL in aqueous solution and the results are shown in Fig. 4. The data for both samplers represents the changes associated with the presence of the electric field on the aerosol particle number density and size distribution in a streamline located near the collector surface (Airpoint) or from the cylindrical shell centerline (ESSA).

These results reveal that the collection efficiency of the

a)
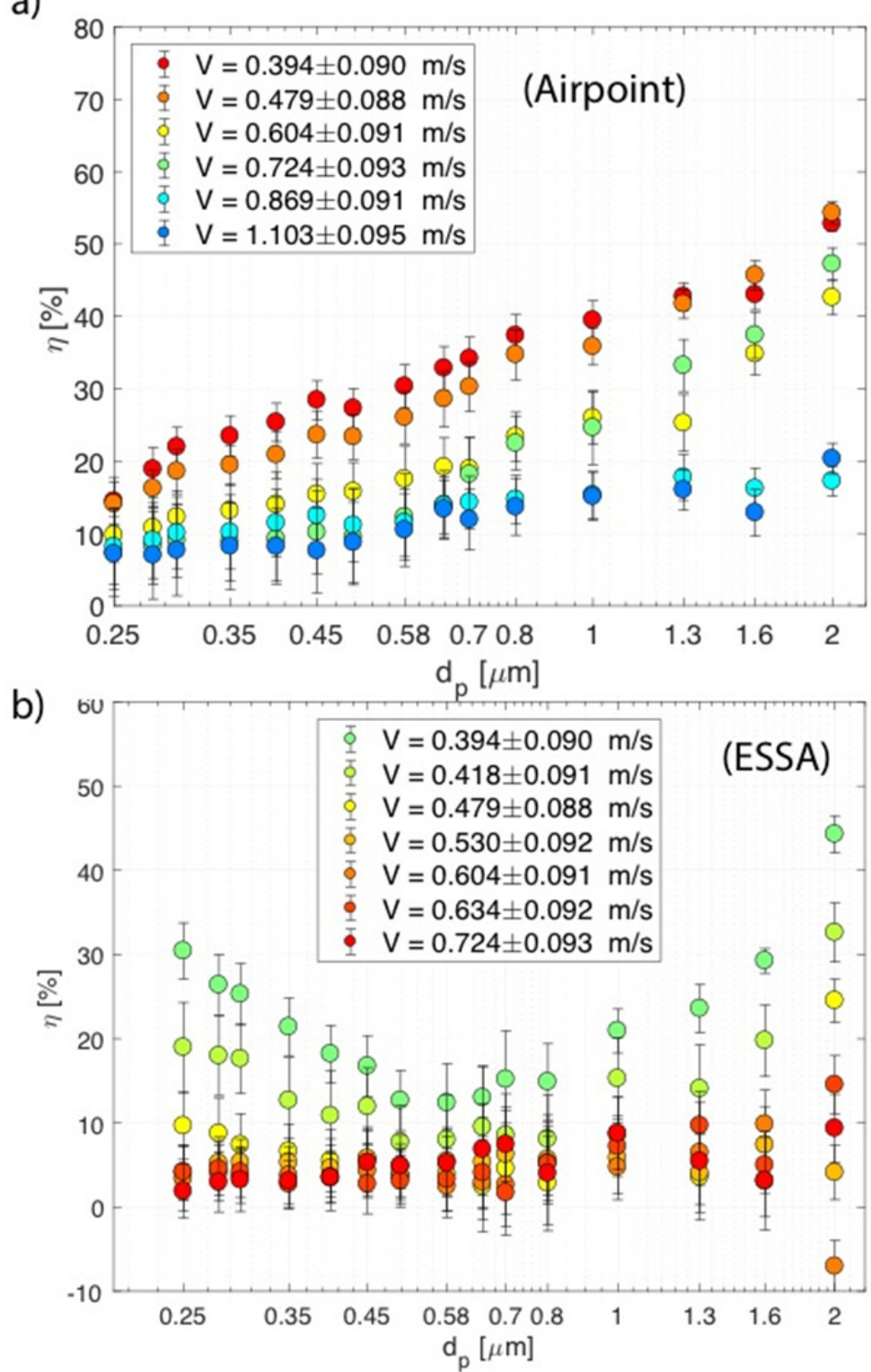

Fig. 4. Collection efficiency for $\mathrm{KCl}$ aerosol particles as function of their size and of the average air flow velocity surrounding the Airpoint (a) and ESSA (b) samplers. The horizontal axis is represented in logarithmic scale. (a) Sampling probe located $3 \mathrm{~mm}$ above and $20 \mathrm{~mm}$ downstream of the collecting surface of the Airpoint, with an electrical potential difference of $17 \mathrm{kV}$ applied between the electrodes. (b) Sampling probe $20 \mathrm{~mm}$ away from the axis of the ESSA's cylindrical shell and $20 \mathrm{~mm}$ downstream its downstream opening, with an electrical potential difference of $17 \mathrm{kV}$. 
Airpoint increases with particle size, as expected from the higher number of ions that can accumulate on larger particles, as shown in Eqs. S(1) and S(2) of the theoretical development included as supplementary material. The collection efficiency for all particle sizes is also shown to decrease when the air bulk velocity is increased, which is also expected from the shorter residence time of particles in the electric field. However, these observations are device-specific as complex flow patterns can be created by the electric field next to the charging needles, significantly affecting the relationship between bulk flow velocity and particle residence time in the field.

Contrary to what was observed for the Airpoint sampler, the results for the ESSA, presented in Fig. 4(b), show a minimum collection efficiency for particles with a size in the range of $0.4 \mu \mathrm{m}$ to $0.8 \mu \mathrm{m}$, for the three lowest air velocities. This behavior can be associated with the presence of the inter-electrode re-circulation zones, as described in Section Air Velocity Profile at the Samplers. Because of their tendency to follow streamlines, the smallest particles are more likely to remain trapped in these regions. Therefore, they have a longer residence time in the electric field compare to larger particles, acquiring more charge, which in turn increases their chance for collection.

Fig. 5(a) shows the collection efficiency at different vertical distance from the Airpoint collector plate and at different radial distance from the axis of the ESSA's cylindrical shell, for a fixed bulk flow velocity of $0.394 \mathrm{~m} \mathrm{~s}^{-1}$. For the Airpoint, the measurements show a clear and monotonic decrease in collection efficiency with increasing

a)

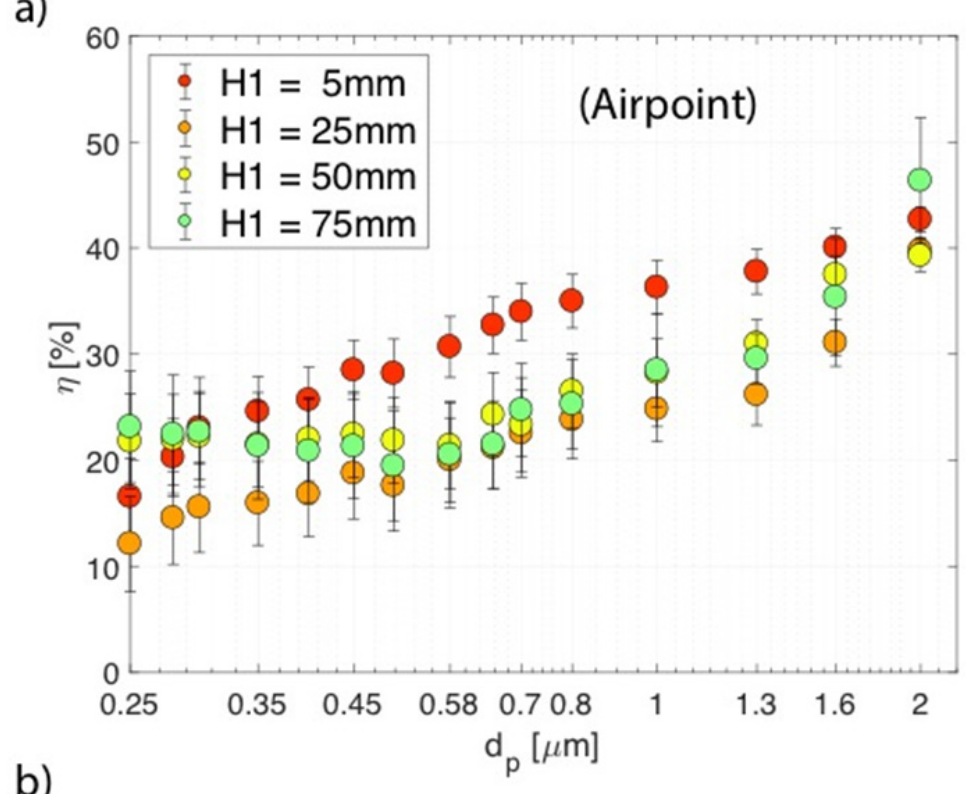

b)

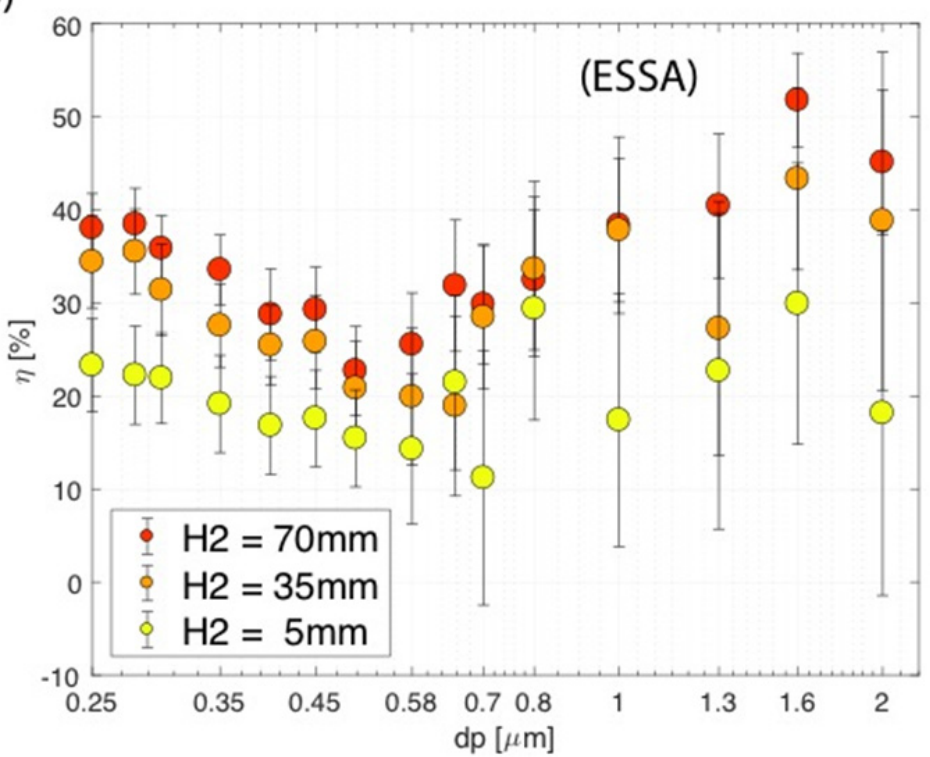

Fig. 5. Collection efficiency of the Airpoint (a) and ESSA (b) samplers as function of particle size and distance from the collector surface (Airpoint, H1) and axis of the shell (ESSA, H2). The horizontal axis is represented with a logarithmic scale. In both cases the velocity is fixed at $0.39 \mathrm{~m} \mathrm{~s}^{-1}$. 
vertical distance relative to the collecting surface, for all particle sizes. At a height of $75 \mathrm{~mm}$, there is approximately zero collection of particles smaller than $<0.70 \mu \mathrm{m}$. This reveals that for this velocity the Airpoint can only capture such small particles when they circulate closer than $75 \mathrm{~mm}$ from its collector. This behavior can be easily understood by the fact that the electrical field weakens with distance from the electrodes, resulting in decreasing accumulation of electrical charges, affecting small particles first as they are more difficult to charge. This information is critical to properly interpret the results provided by this type of passive sampler, as they are often marketed and used assuming that they can provide a representative measure of the aerosols present in a room. For the small particles investigated here this is clearly not the case as the influence of the sampler only extends only a few centimeters from the collector surface, even in low ambient air currents.

The result in Fig. 5(b) shows that the collection efficiency of the ESSA increases with increasing radial distance from the shell center line. This trend is expected because of the arrangement of the ionizing needles, positioned on a ring $35 \mathrm{~mm}$ from the axis of the sampler and aligned with the flow. The presence of re-circulating structure in the interelectrode region, caused by the convergence on the collector of the strong convective stream emanating from the needles, also contribute to this design being able to capture particles from a much bigger cross-sectional area of the flow, compared to the Airpoint.

Moreover, by considering the data presented in Fig. 5 to identify the regions where measurable sampling occurs, the volumetric flow rate of air impacted by the presence of the sampler can be estimated. Assuming that the Airpoint sampler has the same performance over the width of its collecting electrode, when inserted in an air stream flowing at $0.39 \mathrm{~m} \mathrm{~s}^{-1}$ it samples particles in approximately $150 \mathrm{~L} \mathrm{~min}^{-1}$ of air. The same calculation in the ESSA yields a sampled volumetric flow rate of $360 \mathrm{~L} \mathrm{~min}^{-1}$.

\section{Discussion on Electrostatic Sampler Design}

By harnessing simultaneously the ability of electrostatic fields to generate air currents, charge particles and collect them on an electrode, the samplers investigated here can bring highly desirable capabilities to airborne pathogen sampling. The design of such samplers is a young field and the results presented above provide guidelines for the optimization of field and flow geometries. They reveal that to maximize the volumetric flow rate of air that can be treated, field geometry and needle placement is paramount. As demonstrated by the ESSA sampler ability to collect particles initially located much further from the collector compared to the Airpoint, a large number of charging needles distributed around a central collecting electrode is an advantage.

The strength of the air current generated by the field itself is also directly related to the ability of the sampler to collect particles effectively in air currents. As shown in Section Air Velocity Profile at the Samplers, even under moderate bulk flow velocities, the spatial extent of the aerosol sampling performed by the Airpoint is surprisingly limited. It follows that to reliably sample whole rooms this type of device either should be moved around or must rely on external convection to bring particles close to its collection surface. Great care should also be taken to avoid locating such samplers within recirculation bubbles, for instance on top of shelves or in room corners, where the effective volume sampled can be much smaller than the room itself.

By taking these results into consideration, it becomes possible to design electrostatic samplers tailored for specific tasks. For instance, to maximizes collection efficiency for small particles a sampler should aim to create a flow and field geometry that results in the presence of recirculation zones in regions of intense electric field strength. Submicron particles can benefit from the extended time in these recirculation zones exposed to the field to acquire sufficient charge to be collected. In the ESSA design, these recirculation zone were created in the smooth cylindrical shell by the jets of charged air molecules converging towards the central collection electrode. The geometry of axisymmetric samplers could however also be used to create or increase the intensity of recirculation zones, for instance by adding a step change (expansion) in the cross section of a cylindrical shell, slightly downstream of the charging needles.

\section{CONCLUSIONS}

Two electrostatic samplers designed for the capture of bioaerosol particles are investigated to identify the effect of two key parameters on collection efficiency: the size of the particles and the velocity of air currents surrounding the samplers. The results show that for the two designs studied the collection efficiency for all particle sizes decreases as the air bulk velocity increases, as expected from the theory for electrostatic particle charging. The collection efficiency of the planar sampler design investigated (Airpoint) reveals that it effectively samples only the streamlines closer than $7.5 \mathrm{~cm}$ from the collector surface, even in low velocity ambient air currents $\left(0.4 \mathrm{~m} \mathrm{~s}^{-1}\right)$. The collection efficiency of the axisymmetric sampler design (ESSA) exhibits a minimum for particles with a diameter in the range of $0.4 \mu \mathrm{m}$ to $0.8 \mu \mathrm{m}$. Flow field visualizations using PIV associate this unexpected trend with the established convective cells caused by the electric field in the inter-electrode region. The results highlight the importance of measuring velocity fields when assessing the collection efficiency of electrostatic samplers and can assist in the design of geometries that maximizes performance in desired size ranges by carefully tailoring the electrical field and the recirculation zones it creates in the flow.

\section{ACKNOWLEDGMENTS}

The research presented here has received funding from the KTH Linné FLOW Center, the Swedish Research Council (VR) and the European Union's Seventh Framework Programme (FP7) under Grant Agreement No. 604244 (Norosensor). 


\section{SUPPLEMENTARY MATERIAL}

Supplementary data associated with this article can be found in the online version at http://www.aaqr.org.

\section{REFERENCES}

Alonso, C., Raynor, P.C., Davies, P.R. and Torremorell, M. (2015). Concentration, size distribution, and infectivity of airborne particles carrying swine viruses. PLoS One 10: $\mathrm{e} 0135675$.

Baldwin, P.E.J. and Maynard, A.D. (1998). A survey of wind speeds in indoor workplaces. Ann. Occup. Hyg. 42: 303-313.

Bonifait, L., Charlebois, R., Vimont, A., Turgeon, N., Veillette, M., Longtin, Y., Jean, J. and Duchaine, C. (2015). Detection and quantification of airborne norovirus during outbreaks in healthcare facilities. Clin. Infect. Dis. 61: 299-304.

Brown, J.K. and Hovmøller, M.S. (2002). Aerial dispersal of pathogens on the global and continental scales and its impact on plant disease. Science 297: 537-541.

Dybwad, M., Skogan, G. and Blatny, J.M. (2014). Comparative testing and evaluation of nine different air samplers: End-to-end sampling efficiencies as specific performance measurements for bioaerosol applications. Aerosol Sci. Technol. 48: 282-295.

Gerone, P.J., Couch, R.B., Keefer, G.V., Douglas, R., Derrenbacher, E.B. and Knight, V. (1966). Assessment of experimental and natural viral aerosols. Bacteriol. Rev. 30: 576-588.

Hagbom, M., Nordgren, J., Nybom, R., Hedlund, K.O., Wigzell, H. and Svensson, L. (2015). Ionizing air affects influenza virus infectivity and prevents airbornetransmission. Sci. Rep. 5: 11431-11431.

Han, T. and Mainelis, G. (2008). Design and development of an electrostatic sampler for bioaerosols with high concentration rate. J. Aerosol Sci. 39: 1066-1078.

Han, T., Zhen, H., Fennell, D.E. and Mainelis, G. (2015). Design and evaluation of the field-deployable electrostatic precipitator with superhydrophobic surface (FDEPSS) with high concentration rate. Aerosol Air Qual. Res. 15: 2397-2408.

Kettleson, E.M., Schriewer, J.M., Buller, R.M.L. and Biswas, P. (2013). Soft-X-ray-enhanced electrostatic precipitation for protection against inhalable allergens, ultrafine particles, and microbial infections. Appl. Environ. Microbiol. 79: 1333-1341.

Kulkarni, P., Baron, P.A. and Willeke, K. (2011). Aerosol measurement: Principles, techniques, and applications. John Wiley \& Sons, New York, USA.

Ladhani, L., Pardon, G. and van der Wijngaart, W. (2015). A 3D microfluidic cage collector for airborne particles,
The $19^{\text {th }}$ International Conference on Miniaturised Systems for Chemistry and Life Sciences, MicroTAS, Royal Society of Chemistry, pp. 79-81.

Lighthart, B. and Shaffer, B.T. (1997). Increased airborne bacterial survival as a function of particle content and size. Aerosol Sci. Technol. 27: 439-446.

Ning, Z., Sillanpää, M., Pakbin, P. and Sioutas, C. (2008). Field evaluation of a new particle concentratorelectrostatic precipitator system for measuring chemical and toxicological properties of particulate matter. Part. Fibre Toxicol. 5: 15.

Pardon, G., Ladhani, L., Sandström, N., Ettori, M., Lobov, G. and van der Wijngaart, W. (2015). Aerosol sampling using an electrostatic precipitator integrated with a microfluidic interface. Sens. Actuators B 212: 344-352.

Park, J.W., Park, C.W., Lee, S.H. and Hwang, J. (2015). Fast monitoring of indoor bioaerosol concentrations with ATP bioluminescence assay using an electrostatic rod-type sampler. PLoS One 10: e0125251.

Parvaneh, S., Ahlf, E., Elfman, L.H.M., Van HageHamsten, M., Elfman, L. and Nybom, R. (2000). A new method for collecting airborne allergens. Allergy 55: 1148-1154.

Sillanpää, M., Geller, M.D., Phuleria, H.C. and Sioutas, C. (2008). High collection efficiency electrostatic precipitator for in vitro cell exposure to concentrated ambient particulate matter (PM). J. Aerosol Sci. 39: 335-347.

van Leuken, J., Swart, A., Havelaar, A., Van Pul, A., van der Hoek, W. and Heederik, D. (2016). Atmospheric dispersion modelling of bioaerosols that are pathogenic to humans and livestock-A review to inform risk assessment studies. Microb. Risk Anal. 1: 19-39.

Verreault, D., Moineau, S. and Duchaine, C. (2008). Methods for sampling of airborne viruses. Microbiol. Mol. Biol. Rev. 72: 413-444.

Walser, S.M., Gerstner, D.G., Brenner, B., Bünger, J., Eikmann, T., Janssen, B., Kolb, S., Kolk, A., Nowak, D., Raulf, M., Sagunski, H., Sedlmaier, N., Suchenwirth, R., Wiesmüller, G., Wollin, K.M., Tesseraux, I. and Herr, C.E.W. (2015). Evaluation of exposure-response relationships for health effects of microbial bioaerosols A systematic review. Int. J. Hyg. Environ. Health 218: 577-589.

Willeke, K., Grinshpun, S.A., Ulevicius, V., Terzieva, S., Donnelly, J., Stewart, S. and Juozaitis, A. (1995). Microbial stress, bounce and re-aerosolization in bioaerosol samplers. J. Aerosol Sci. 26: S883-S884.

Received for review, June 6, 2018 Revised, September 15, 2018 Accepted, September 29, 2018 